\title{
AI in Healthcare: medical and socio-economic benefits and challenges
}

\author{
Mohammed Yousef Shaheen
}

\begin{abstract}
The objective of this research was to investigate benefits and challenges of $\mathrm{Al}$ in healthcare. We divided the benefits into two subcategories: benefits related to the medical domain, and the benefits related to economic and social lives domain. The findings are: 1) Smart data inclusion contributes significantly and help to improve decision-making quality. 2) Surgical robots have improved the precision and predictability of the surgery. 3) Intraoperative guidance via video pictures and communication systems has proven to be beneficial, particularly in situations when there is a poor access to clinics, travel limitations, or pandemic. 4) Sentiment analysis analyzes, interprets, and responds to verbal expressions of human emotions. 6) Data scientists have been able to create algorithms that can comprehend human feeling from written text with unique combination of NLP and sentiment analysis. 7) Al could re-balance a clinician's workload, providing them more time to connect with patients and thereby improve care quality. The major challenges are: 1) the data reflects sometimes inherent biases and disparities in the healthcare system. 2) The demand for huge datasets incentivizes developers to acquire data from a large number of patients. Some patients may be worried that this data collection would infringe on their confidentiality. 3) Al systems may occasionally be incorrect, resulting in patient damage or other health-care issues. It is not assumed that a new technology will always be good; it has the potential to be detrimental. There are some improvements that benefit and there are some challenges that may harm, and these challenges must be responded by future research.
\end{abstract} Keywords: Al, Bias, Cost minimization, 


\section{Introduction}

Healthcare systems are on the verge of collapsing unless massive structural and transformational changes are made. Health systems also require a larger staff, but the World Health Organization estimates that despite the global economy creating 40 million additional health-sector employment by 2030 , there will still be a 9.9 million physician, nurse, and midwife shortage during the same time period (WHO, 2016). Not only is it critical to educate and recruit more health care providers, but it is also critical to ensure that their time is spent where it adds the greatest value-caring for patients.

Artificial intelligence (AI), which is based on automation, has the potential to change healthcare and assist tackle some of the crucial issues. Al is a computer program's capacity to execute tasks or thinking processes that we typically identify with intelligence in a human being. Al has the potential to improve patient outcomes as well as the efficiency and productivity of healthcare delivery (Shaheen, 2021b). It can also improve the day-to-day lives of healthcare providers by allowing them to spend more time caring for patients, hence improving staff morale and retention. It may even be able to speed up the development of life-saving therapies.

The Automation system is at the center of the healthcare industry's innovation. With the ratio of healthcare workers to patients on the decline, robotics and artificial intelligence in healthcare are nonetheless filling the hole, and providing accuracy and precision that eliminates the possibility of human error.

There will be few phases of Al scaling in healthcare over time, based on existing solutions and concepts in the process (Govindasamy, 2019a). First, approaches are likely to focus on the mundane, repetitive, and primarily administrative procedures that take up a significant amount of time for doctors and nurses, hence improving healthcare operations and promoting adoption (Govindasamy, 2019b). There are Al solutions based on imaging in this initial phase, which are already in practice in disciplines like radiology and ophthalmology.

Secondly, as patients take greater control of their care, we expect additional Al solutions to assist the transition from clinic-based to home-based treatment, such as remote patient monitoring, Alpowered alarm systems, or virtual aides (Arora, 2020). This phase might also include expanded usage of NLP solutions in hospitals and at home, as well as increased Al application across a broader range of specialties, such as oncology, and neurology, where progress is already being made. This will necessitate a greater integration of Al into healthcare operations, which will necessitate the active participation of professional organizations and providers (Apell \& Eriksson, 2021). To use old technology efficiently in new contexts, it will also require well-designed and integrated solutions. A mix of technological breakthroughs and culture change and competence building within enterprises will drive this scaling up of Al adoption (Shaheen, 2021a).

We can anticipate to witness more Al applications in clinical settings based on empirical supports from clinical trials, with a greater emphasis on enhanced and expanded clinical decisionsupport techniques in a sector that has managed to learn from previous efforts to implement such techniques into clinical practice and has adjusted its mind-set, culture, and skills. We expect Al to play a significant role in the healthcare value stream, from how we learn to how we explore and give care to how we enhance population health. Integration of bigger data sets across companies, robust governance to continuously enhance data quality, and increased faith from organizations, practitioners, and patients in both the Al technologies and the ability to manage the associated risks will be key prerequisites for Al to fulfill its maximum potential in healthcare. 


\section{Medical benefits}

\subsection{Better data-driven decision to improve the healthcare system}

Identifying a decision, acquiring information, and evaluating possible remedies are all steps in the decision-making process. By collecting important information and identifying options, a staged decision-making procedure can assist you in making more careful, considered decisions.

The quality of decision-making in today's digitalized healthcare environment is heavily reliant on the availability and accuracy of the underlying data (Madsen, 2014). In healthcare, where clinical decision-makers encounter many hurdles and problems throughout the patient pathway, smart data inclusion can contribute significantly and help to improve decision-making quality. Because data is unavailable or too large to examine, information is overlooked, or recommendations are disregarded, complex judgments in healthcare may fail, resulting in inefficient and costly processes and impaired clinical outcomes (Guo et al., 2020.) (Chonghui Guo \& Chen, 2019). Al utilize the following: a) Patient data to aid clinical decision-making b) Data from hospitals to aid operational decision-making, and c) Data about patients and hospitals to aid in consumer decision-making

\subsection{Al assists in improving surgery}

Artificial intelligence offers a multitude of applications in the domains of medicine and dentistry. Surgical robots have improved the precision and predictability of the surgery. Robotic surgery is used extensively in many areas of surgery, including oral and maxillofacial surgery. Bioprinting, diabetic retinopathy, spine imaging, and radiology are among the other applications (Hashimoto et al., 2020) (Zhou et al., 2019). Augmented reality, on the other hand, superimposes a computer-generated image on a patient's vision of the real world to create a consolidated view of the surgical field.

Al-powered Tele-surgical approaches have enabled remote surgery and senior surgeon oversight of surgeons in the surgery room. Intraoperative guidance via video pictures and communication systems has proven to be beneficial, particularly in situations when there is a poor access to clinics, travel limitations, or pandemics (Fekri et al., 2018). Al and AR-powered surgical mentorship are becoming a viable alternative for many surgeons as less invasive surgical techniques become more common (Hashimoto et al., 2018). Experienced surgeons can provide real-time counseling on the best incision or equipment to use on the operator's screen, guiding the surgeon through the surgical operation. The workflow of a surgeon can also be inspected using data and computer vision techniques.

Robots assist in the analysis of the patient's medical information prior to surgery in order to direct the surgical instruments during the procedure. Due to the minimally invasive nature of robotassisted surgery, it has been proven to dramatically minimize the length of patient stay in the hospital (Prabu et al., 2014). Robots can also utilize Al to offer newer surgical approaches based on a patient's previous surgery history. An eye surgery using the Da Vinci surgical robot allows physicians to conduct complex procedures with more control (Hockstein et al., 2005).

\subsection{Supports mental health}

Artificial intelligence (AI) is acquiring popularity in mental health treatment, as patients choose ease and immediate feedback (Luxton, 2016). Because our emotional and mental well-being is mostly expressed through language, psychiatric experts have traditionally depended on therapeutic discourse and patient narrative to measure mental health. Recent advances in Artificial 
Intelligence have provided new perspectives into the field by allowing technology to deduce emotional meaning from a wider range of data sources (Lovejoy, 2019).

Computational languages and sentiment assessment have played an important role in this process. Natural Language Processing (NLP) is a technique used in computer linguistics to develop computational algorithms that can analyze raw human language input. Sentiment analysis is a branch of artificial intelligence that analyzes, interprets, and responds to verbal expressions of human emotions (Graham et al., 2019) (Habermann, 2021b). Data scientists have been able to create algorithms that can comprehend human feeling from written text with unique combination of NLP and sentiment analysis (Habermann, 2021a). These models are currently being utilized in medicine to provide detailed information on a patient's emotional and psychological well-being.

NLP models have been used to detect suicidal thoughts in clinical notes, forecast suicide risk online, and mine for psychiatric self-disclosure on Twitter in recent years (Le Glaz et al., 2021). Both personal patient care and broader public health policy can benefit from these models. NLP algorithms that efficiently map behavioral health diseases across the U. S., associating with public health information from the Centers for Disease Control and Prevention, are examples of population level applications (CDC). Researchers have proven great accuracy in forecasting which moms may experience postnatal depression employing their online information on an individual level (Luxton, 2014) (Luxton, 2016).

\section{Economic and social benefits: Cost and energy saving}

While we are not quite at the level where autonomous robots are doing every work there is good evidence that Al can assist control healthcare expenses in a variety of ways.

\subsection{Elimination of expenditures associated with post-treatment problems}

In today's environment, being able to properly and precisely harness the power of data allows for more efficient decision-making in almost every industry. Healthcare is no exception. Large quantities of data will emerge accessible for analysis by Al-powered systems as healthcare providers move toward an uniform format for recording patient outcomes. These systems will be able to analyze result patterns following treatment and find appropriate therapies based on patients' profiles. As a result, Al facilitates clinical decision-making and guarantees that the appropriate interventions and therapies are tailored to each patient, resulting in a more individualized approach to care. The immediate result will be a dramatic increase in outcomes, resulting in the elimination of expenditures associated with post-treatment problems - one of the major cost drivers in most healthcare ecosystems around the world (Le Nguyen \& Do, 2019) .

\subsection{Cost cutting through early diagnostics}

Al-enabled devices can execute repetitive, straightforward activities more accurately, including as CT scan processing and certain tests, decreasing physician mistakes and facilitating timely diagnosis and action before situations become serious. Al has showed big precision and faster capability than humans in analyzing and deciphering mammograms, allowing for considerably earlier identification of breast cancer than humans. The recognition of vertebral fractures - an early sign of incipient osteoporosis that is frequently missed by human diagnosis - can significantly decrease the expense of this condition to health services in cases like osteoporosis 


\subsection{Cost minimization with enhanced clinical trials}

Al has the ability to accelerate the creation of life-saving pharmaceuticals, saving billions of dollars in expenses that can be passed on to health-care systems. Al powered program that simulates and analyzes millions of potential treatments to forecast their efficacy against Ebola, saving money and, most crucially, lives. Utilizing biomarker monitoring frameworks - biomarkers enable for gene-level illness detection - and a large quantity of patient data points, that can be analyzed in seconds from a sample of blood applying at-home devices - Al can optimize medication development in clinical trials (Beck et al., 2020).

\subsection{Patient empowerment}

Artificial intelligence has the ability to actually empower us as people to make better health decisions. Wearable technology is already being used by a large number of people all over the world to collect common data such as sleep patterns and heart rate. Using machine learning to analyze this data could alert people who are at risk of developing some diseases long before they become serious. Patients with certain chronic diseases may be able to better control their disease and live healthier lives with the use of mobile apps that provide granular-level patient profile information. All of this has the potential to result in healthier populations and lower total costs (Ichikawa et al., 2016) (Vollmer et al., 2020).

\section{Challenges}

While Al has a lot of potential benefits, it also has a number of drawbacks:

\section{1 bias}

In health-care $\mathrm{Al}$, there are dangers of prejudice and inequality. Al systems learn from the information they've been given and can absorb biases from that data. For example, if Al data is primarily collected in academic medical institutions, the subsequent Al systems will learn less about - and hence treat patients from communities that do not often visit academic medical centers and thus will treat them less effectively. Similarly, when speech-recognition Al systems are employed to transcribe encounter notes, the Al may function worse if the provider belongs to a race or gender that is underrepresented in the training data.

Even if Al systems are trained on reliable, representative data, there may be issues if the data reflects inherent biases and disparities in the healthcare system. An Al system learning from healthcare records might learn to recommend lower painkiller doses to African-American patients, despite the fact that this decision is based on systemic bias rather than biological reality (Lee, 2021). Al systems that allocate resources could aggravate inequality by allocating limited resources to patients who are deemed less desirable or lucrative by health systems for a number of reasons.

\subsection{Missteps and accidents.}

The most obvious danger is that Al systems will occasionally be incorrect, resulting in patient damage or other health-care issues. A patient could be damaged if an Al system prescribes the wrong treatment, fails to detect a tumor on a radiological exam, or assigns a medical bed to one person over another because the Al system incorrectly projected which patient would gain more. Of 
all, many injuries occur in the health-care system nowadays owing to medical error, even without the use of Al. For at least two reasons, Al errors are possibly distinct. For starters, injuries caused by software may elicit different reactions from patients and caregivers than injuries caused by human mistake. Second, if Al systems become more widely used, a flaw in one Al system might lead to thousands of patients being wounded, rather than the small patients injured by a single provider's error.

\subsection{Concerns about privacy.}

Another set of dangers arises when it comes to privacy. The demand for huge datasets incentivizes developers to acquire data from a large number of patients. Some patients may be worried that this data collection would infringe on their confidentiality, and lawsuits have been filed as a result of data sharing between huge health institutions and Al companies. Al could also compromise privacy by predicting personal information of patients even if the algorithm has never been given that information (Marwan et al., 2018). (In fact, this is frequently the purpose of Al in health care.) For example, an Al system might be able to tell if someone has Parkinson's disease just on the shaking of a computer mouse, even if that individual has never told anybody else (or did not know). Patients may see this as a breach of their privacy, particularly if the Al system's conclusions are made public to third parties like banks or life insurance firms (van der Schaar et al., 2021).

\subsection{Data accessibility}

Large volumes of data from sources like electronic health information, pharmacy documents, insurance claims documents, or consumer-generated data like activity trackers or purchase history are needed to train Al algorithms. However, health statistics can be difficult to come by. Data is frequently dispersed across multiple platforms. Aside from the variation noted above, patients frequently see different physicians and change insurance carriers, resulting in data being fragmented across multiple systems and formats. This fragmentation raises the danger of inaccuracy, reduces the comprehensiveness of datasets, and raises the cost of data collection-all of which restrict the types of entities that might create successful health-care Al (Hu et al., 2015).

\section{Conclusion}

Artificial intelligence can not only aid in the development of useful pharmaceuticals, but also improve the efficacy of current ones after they have been developed. Although Al systems in healthcare is currently restricted, the medical and economical benefits are too great to overlook. High-cost sectors will become possibilities for forward-thinking healthcare businesses to employ breakthrough technology and preserve their competitive superiority over their counterparts. These examples only scratch the surface of what is conceivable when Al's full potential is applied to healthcare delivery. The possibilities cannot be overstated or understated, and collaboration between government and private domain industry players is critical to realizing this potential. The growing expense of healthcare will remain to be a hot topic among healthcare stakeholders as global communities live longer and the frequency of chronic disease rises. Perhaps it's time to enlist the help of the machines.

Regardless of how exciting these technologies are, they must be seen in the context of our current healthcare change. The incorporation of smart health into medicine is causing significant changes in the healthcare industry, and the choices we take now will have far-reaching implications for patient 
care in the future. Researchers and developers are now developing these tools with the idea that current medical practice is the main framework,' despite the field's lengthy history of discriminatory practices, prejudices, and medical errors.

\section{References}

Apell, P., \& Eriksson, H. (2021). Artificial intelligence (Al) healthcare technology innovations: the current state and challenges from a life science industry perspective. Technology Analysis \& Strategic Management, 1-15.

Arora, A. (2020). Conceptualising artificial intelligence as a digital healthcare innovation: an introductory review. Medical Devices (Auckland, NZ), 13, 223.

Beck, J. T., Rammage, M., Jackson, G. P., Preininger, A. M., Dankwa-Mullan, I., Roebuck, M. C., Torres, A., Holtzen, H., Coverdill, S. E., \& Williamson, M. P. (2020). Artificial intelligence tool for optimizing eligibility screening for clinical trials in a large community cancer center. JCO Clinical Cancer Informatics, 4, 50-59.

Fekri, P., Setoodeh, P., Khosravian, F., Safavi, A. A., \& Zadeh, M. H. (2018). Towards deep secure telesurgery. Proceedings of the International Conference on Scientific Computing (CSC), 81-86.

Govindasamy, S. P. (2019a). Scaling Innovations in Healthcare: A Multi-Method Analysis of Facilitators and Barriers of Innovation Adoption in Hospitals. Temple University.

Govindasamy, S. P. (2019b). Scaling Innovations in Healthcare. Temple University. Libraries.

Graham, S., Depp, C., Lee, E. E., Nebeker, C., Tu, X., Kim, H.-C., \& Jeste, D. V. (2019). Artificial intelligence for mental health and mental illnesses: an overview. Current Psychiatry Reports, 21(11), 1-18.

Guo, C, Engineering, J. C.-J. of S. S. and S., \& 2019, undefined. (n.d.). Big data analytics in healthcare: data-driven methods for typical treatment pattern mining. SpringerPaperpile. Retrieved September 23, 2021, from https://link.springer.com/article/10.1007/s11518-019-5437-5

Guo, Chonghui, \& Chen, J. (2019). Big Data Analytics in Healthcare: Data-Driven Methods for Typical Treatment Pattern Mining. Journal of Systems Science and Systems Engineering, 28(6), 694714. https://doi.org/10.1007/S11518-019-5437-5

Habermann, J. (2021a). Language and psycho-social well-being.

Habermann, J. (2021b). Psychological impacts of COVID-19 and preventive strategies: A review.

Hashimoto, D. A., Rosman, G., Rus, D., \& Meireles, O. R. (2018). Artificial intelligence in surgery: promises and perils. Annals of Surgery, 268(1), 70.

Hashimoto, D. A., Ward, T. M., \& Meireles, O. R. (2020). The role of artificial intelligence in surgery. Advances in Surgery, 54, 89-101.

Hockstein, N. G., Nolan, J. P., O'Malley Jr, B. W., \& Woo, Y. J. (2005). Robotic microlaryngeal surgery: a technical feasibility study using the daVinci surgical robot and an airway mannequin. The Laryngoscope, 115(5), 780-785.

Hu, J., Perer, A., \& Wang, F. (2015). Data driven analytics for personalized healthcare. Healthcare Information Management Systems: Cases, Strategies, and Solutions: Fourth Edition, 529-554. https://doi.org/10.1007/978-3-319-20765-0_31

Ichikawa, D., Saito, T., Ujita, W., \& Oyama, H. (2016). How can machine-learning methods assist in virtual screening for hyperuricemia? A healthcare machine-learning approach. Journal of 
Biomedical Informatics, 64, 20-24.

Le Glaz, A., Haralambous, Y., Kim-Dufor, D.-H., Lenca, P., Billot, R., Ryan, T. C., Marsh, J., Devylder, J., Walter, M., \& Berrouiguet, S. (2021). Machine learning and natural language processing in mental health: Systematic review. Journal of Medical Internet Research, 23(5), e15708.

Le Nguyen, T., \& Do, T. T. H. (2019). Artificial intelligence in healthcare: A new technology benefit for both patients and doctors. 2019 Portland International Conference on Management of Engineering and Technology (PICMET), 1-15.

Lee, E. (2021). How do we build trust in machine learning models? Available at SSRN 3822437.

Lovejoy, C. A. (2019). Technology and mental health: the role of artificial intelligence. European Psychiatry, 55, 1-3.

Luxton, D. D. (2014). Artificial intelligence in psychological practice: Current and future applications and implications. Professional Psychology: Research and Practice, 45(5), 332.

Luxton, D. D. (2016). An introduction to artificial intelligence in behavioral and mental health care. In Artificial intelligence in behavioral and mental health care (pp. 1-26). Elsevier.

Madsen, L. (2014). Data-driven healthcare: how analytics and BI are transforming the industry. https://books.google.com/books?hl=en\&lr=\&id=De_IBQAAQBAJ\&oi=fnd\&pg=PR13\&dq=datadriven+decision+healthcare\&ots=IEbwt52_Au\&sig=QN7m-si9scdFdmQ6Rx5c9W6nqpo

Marwan, M., Kartit, A., \& Ouahmane, H. (2018). Security enhancement in healthcare cloud using machine learning. Procedia Computer Science, 127, 388-397.

Organization, W. H. (2016). Health workforce requirements for universal health coverage and the sustainable development goals. (human resources for health observer, 17).

Prabu, A. J., Narmadha, J., \& Jeyaprakash, K. (2014). Artificial Intelligence Robotically Assisted Brain Surgery. Artificial Intelligence, 4(05).

Shaheen, M. Y. (2021a). Adoption of machine learning for medical diagnosis.

Shaheen, M. Y. (2021b). Applications of Artificial Intelligence (AI) in healthcare: A review.

van der Schaar, M., Alaa, A. M., Floto, A., Gimson, A., Scholtes, S., Wood, A., McKinney, E., Jarrett, D., Lio, P., \& Ercole, A. (2021). How artificial intelligence and machine learning can help healthcare systems respond to COVID-19. Machine Learning, 110(1), 1-14.

Vollmer, S., Mateen, B. A., Bohner, G., Király, F. J., Ghani, R., Jonsson, P., Cumbers, S., Jonas, A., McAllister, K. S. L., \& Myles, P. (2020). Machine learning and artificial intelligence research for patient benefit: 20 critical questions on transparency, replicability, ethics, and effectiveness. $B m j, 368$.

Zhou, X.-Y., Guo, Y., Shen, M., \& Yang, G.-Z. (2019). Artificial intelligence in surgery. ArXiv Preprint ArXiv:2001.00627. 